\title{
A novel method for evaluating sun visible light protection factor and pigmentation protection factor of sunscreens
}

This article was published in the following Dove Press journal:

Clinical, Cosmetic and Investigational Dermatology

\author{
Sérgio Schalka' \\ Marcelo de Paula Corrêa ${ }^{2}$ \\ Leticia Yumi Sawadal \\ Camila C Canale ${ }^{3}$ \\ Thayna $\mathrm{N}$ de Andrade ${ }^{4}$ \\ 'Department of Dermatology, Medcin \\ Clinical Research Center, Osasco, Brazil; \\ ${ }^{2}$ Institute of Natural Resources, Federal \\ University of Itajubá, Itajubá, Brazil; \\ ${ }^{3}$ Department of Pharmacy, Medcin \\ Clinical Research Center, Osasco, Brazil; \\ ${ }^{4}$ Department of Photoprotection, Medcin \\ Clinical Research Center, Osasco, Brazil
}

Correspondence: Sérgio Schalka Department of Dermatology, Medicine Clinical Research Center, Rua Atílio Delanina 178, Osasco 06023-000, SP, Brazil

Tel +55 II 36542054

Email sergio.schalka@medcin.com.br
Introduction: The role of visible light (VL) in the process of skin pigmentation by solar radiation has been demonstrated. Sunscreens with the presence of pigments, particularly iron oxide (IO), have a greater protective effect against VL and are, therefore, highly recommended for prevention and treatment of hyperpigmentation disorders. This study aimed to evaluate 33 sunscreen formulations, and through spectrophotometric measurements, proposes new methods to evaluate the VL protection factor and the pigmentation protection factor.

Methods: 33 sunscreen formulations marketed in Brazil were evaluated, including 17 products containing pigments and 16 products with no pigments. Spectrophotometric measurements were taken to determine solar VL protection factor and the pigmentation protection factor, based on the absorption curve of the product, the pigmentation action spectrum and sun spectrum.

Results: Sunscreen with pigments presented a higher solar VL protection factor and pigmentation protection factor compared to products without pigment. The statistical analysis showed a strong correlation between the solar VL protection factor and the integral of the absorption curve in the VL range and the transmittance reduction (\%) in the same range. The correlation between the VL protection factor and the pigmentation protection factor was also demonstrated.

Conclusion: The VL protection factor and the pigmentation protection factor showed to be adequate metrics to estimate the effectiveness of sunscreens in the prevention of the pigmentary effect of solar VL and the pigmentary effect of the complete spectrum of immediate pigmentation.

Keywords: visible light, sunscreening agents, sun protection factor, skin pigmentation

\section{Introduction}

Visible Light (VL) corresponds to the range of radiation visible to the human eye in a spectrum ranging from violet $(400 \mathrm{~nm})$ to red $(780 \mathrm{~nm})$, and until recently it was believed that VL had no significant photobiological effect. ${ }^{1}$ However, this notion has been challenged by reports of the influence of the VL on several biological effects, such as erythema, pigmentation, thermal damage ${ }^{2}$ and production of Reactive Oxygen Species (ROS). ${ }^{3}$ In addition, several pathologies such as solar urticaria, ${ }^{4}$ chronic actinic dermatitis, ${ }^{5}$ porphyria, ${ }^{6}$ polymorphic light eruption, actinic prurigo and other phototoxic and photoallergic reactions ${ }^{2}$ have been related to the VL action.

There are several studies correlating VL exposure with the induction of cutaneous pigmentation, both transient and persistent, independent of the UVR 
component and with dose-dependent effect. ${ }^{7-10}$ It has been shown by confocal microscopy and histopathological analysis that $\mathrm{VL}$ is able to induce the redistribution of melanin granules from the basal layer to more surface layers of the epidermis; this effect is sustained for at least 2 weeks. ${ }^{9}$

The different wavelengths in VL present different biological actions, with the band with the highest energy (named high energy VL/HEVL, or simply blue light) having the greatest photobiological action. In their study, Pathak ${ }^{11}$ suggested that the peak of the immediate pigment darkening (IPD) response would occur between 380 and $500 \mathrm{~nm}$. Duteil et $\mathrm{al}^{12}$ compared the in vivo induced pigmentation by wavelengths in the blue/violet $(415 \mathrm{~nm})$ versus red light $(630 \mathrm{~nm})$ band and observed intense and lasting pigment formation in the blue light band, with a clear dose-effect relationship, whereas red light failed to induce pigmentation.

The action of the VL on the skin can also vary according to the characteristics of the skin. Mahmoud ${ }^{9}$ observed more intense and lasting erythema and pigmentation formation in individuals with darker phototypes (V-VI), whereas lighter phototypes (II) were not able to produce any pigmentation. This study contrasts with the observations of Porges ${ }^{13}$ and Duteil ${ }^{12}$ (2014), successfully in inducing pigmentation in lighter phototypes.

More recently, Randhawa et $\mathrm{al}^{10}$ have demonstrated that the pigmentation process resulting from the VL is a photoadaptive process, and therefore is accentuated by successive exposures.

Rosen $^{14}$ demonstrated by in vivo method through a monochromator and by reflectance spectroscopy, the spectra of IPD formation between the UVA and VL varies. According to the study, the production of pigmentation with radiation above $470 \mathrm{~nm}$ would be negligible.

Kohli et $\mathrm{al}^{15}$ irradiated 10 subjects on their backs using two light sources, pure VL and VL+UVA1, and the authors suggested a synergistic relationship between VL and UVA1 and emphasized the need for developing means of photoprotection against VL.

The protection offered by conventional sunscreens, with no pigments in the formula, has not been shown to be effective in reducing VL transmission to the skin and does not contribute to the treatment of pigmentary disorders. ${ }^{1}$

The availability of sunscreens containing pigments, particularly iron oxide, has revolutionized the treatment and prevention of pigmentary disorders, precisely by providing protection (essentially by reflection) within the long UVA and high energy VL range. ${ }^{1}$ The use of colored sunscreens (UVR+VIS) showed to be more efficient in avoiding melasma recurrence compared to equivalent UV protection sunscreens, but without protection against the VL. ${ }^{16,17}$ When associated with bleaching therapy in patients with melasma, sunscreens with VL protection enhanced the response, with a greater reduction of epidermal melanin content and number of mast cells in the dermis. ${ }^{18}$

For adequate reduction of the VL transmission to the skin, the sunscreen should have in its formulation ingredients capable of reflecting this specific range of sunlight and therefore, rendering it also visible to the human eye. ${ }^{19}$

Inorganic sunscreens, such as zinc oxide and titanium dioxide, act by absorption, reflection and scattering of the solar radiation. The spectrum of action and the relation between absorption and reflection of these elements are related to particle size. ${ }^{20}$ Minor particles, in micro or nanoscale, act essentially through absorption, more specifically within ultraviolet radiation, whereas larger particles also act by reflection, and therefore, with a greater spectrum of performance in the VL range, but, on the other hand, presenting a whitish appearance on the skin. ${ }^{21,22}$ It is important to note that most of the sunscreens available on the market today contain micronized form of these particles, because they have better cosmetic acceptability, ${ }^{20}$ but their reduced size compromises the protection provided against VL. Iron oxide (IO) is a widely used particle in the cosmetic industry, as a pigment in makeup and sunscreens. From the regulatory point of view, it is not classified as a UVR filter, but its excellent photoprotective action in the VL range has motivated its use in tinted sunscreens, recommended in the treatment and prevention of pigmentary disorders. ${ }^{23}$

Despite studies demonstrating the importance of protection against VL, there is no standardized method to evaluate the degree of protection afforded by sunscreen products available on the market.

In a previously published study, ${ }^{19}$ our group compared the absorption/transmission profile of 20 sunscreens marketed in Brazil, with different SPF (sun protector factor) values, being 12 sunscreens with color and 8 sunscreens without color. The results showed that only colored products could significantly reduce energy transmission within the VL range.

In this study, we also showed that the SPF value is not related to the absorptive efficacy in the VL range, the latter is related to the opacity of the product, and therefore in the ability to reflect the VL. Finally, the study proposed a 
method for quantifying protection against visible light by determining the integral of the absorption area of the product determined by spectrophotometry in the high energy VL range (400-450 $\mathrm{nm}$ ). This measure showed a strong correlation with the color and opacity characteristics of sunscreens tested, with more opaque and pigmented products being more effective in protection against VL. ${ }^{19}$

Subsequently, following our study, we present another study, ${ }^{23}$ comparing the reduction of transmittance (\%) in the high energy VL range (HEVL) of 11 sunscreens and demonstrated that products with IO had a transmittance reduction (\%) in this wavelength range not less than $75 \%$, whereas sunscreens without IO presented transmittance reduction (\%) not exceeding $51 \%$.

Duteil et $\mathrm{al}^{12}$ proposed an in vivo method to determine protection against VL using a light source (solar simulator) with daily exposure for 5 days and determining the colorimetric variation of the pigmentation intensity, determined by the Individual Typological Angle. In a more recent study, Ruvolo ${ }^{24}$ promoted the VL exposure in melanocompetent patients (Phototypes IV-VI) and suggested a method to measure the VL protection factor, similar to the SPF in vitro, using a mathematical model similar to that proposed by Sayre et $\mathrm{al}^{25}$.

\section{Purpose}

The aim of this study was to determine a new method to evaluate the effectiveness of sunscreens in reducing solar VL transmission through spectrophotometry. In addition, using concepts proposed by Sayre ${ }^{25}$ and Rosen, ${ }^{14}$ we propose a new method for evaluating the effectiveness of sunscreens by determining the VL Protection Factor (PF-VIS) and the determination of the Pigmentation Protection Factor (PPF), the latter, incorporating the UVA and the VL range.

\section{Methods}

The study compared different sunscreens in the Brazilian market, with respect to spectrophotometric absorption data within the range of $290-450 \mathrm{~nm}$, with particular interest in the range of $320-450 \mathrm{~nm}$.

The study was carried out in the photoprotection laboratory of Medcin Instituto da Pele, a private center of dermatology and clinical research located in the city of Osasco, in the São Paulo Area, from June 2018 to January 2019.

\section{Products studied}

A total of 33 sunscreens marketed in Brazil were selected, with formulation and composition of UVR filters containing different combinations of organic and inorganic filters. Seventeen products contained iron oxide (pigments) in their formulation and sixteen products contained no pigments. The presence of iron oxide and the labeling value of the SPF are shown in Table 1, together with the results of the study.

\section{Materials and equipment Spectrophotometry equipment with integration sphere}

The UVR Transmittance Analyzer UV-2000 (Labsphere ${ }^{\circledR}$, New Hampshire - USA) emits irradiation through a $10 \mathrm{~W}$ xenon lamp in the $250-450 \mathrm{~nm}$ range at wavelength ranges of $1 \mathrm{~nm}$, detected by the spectrographs.

\section{Multiport solar simulator 60I-300W (solar light, glenside, PA - USA)}

The Solar Simulator consists of a $300 \mathrm{~W}$ Xenon lamp and set of filters (WG320, $11.3 \mathrm{~mm}$ diameter and UG11, $11.3 \mathrm{~mm}$, coated) that restrict irradiation to the desired spectrum. This allows the spectral radiation on the surface of exposure to UVR radiation to be as similar as possible to the radiation at ground level under a zenith sun (standard ISO 24443:2012). ${ }^{26}$

\section{Polymethylmethacrylate plates (PMMA)}

The plates used (Schonberg, Germany), with a rough surface of $6 \mu \mathrm{m}(50 \mathrm{mmx} 50 \mathrm{~mm})$ and Roughness Ra between 4.54 and $5.17 \mu \mathrm{m}$, meet the requirements of standard ISO 24443:2012. ${ }^{26}$

\section{Procedures}

The procedures related to the use of the polymethylmethacrylate (PMMA) plates, the application of the products and reading followed the concepts present in standard ISO 24443:2012 $2^{26}$ for determining the protection factor UVA in vitro.

\section{Spectrophotometric evaluation}

For the study, each product was applied to four PMMA plates in the amount of $1.3 \mathrm{mg} / \mathrm{cm}^{2}$ over the rough surface.

The amount and the method of application of the product in the substrate are critical for the fidelity and reproducibility of the test, and therefore all procedures were performed by the same researcher, with experience in the application method and following the procedures were determined by standard ISO 24443:2012. ${ }^{26}$ 
Table I List of sunscreens evaluated with respective classification by presence of iron oxide in the formulation (IO), labelled Sun Protection Factor (SPF), Solar VL Protection Factor (PF-VIS), Pigmentation Protection Factor (PPF), UVA Protection Factor (PF-UVA), transmittance reduction (TR\%) and Integral area in the VL range (IVis)

\begin{tabular}{|c|c|c|c|c|c|c|c|}
\hline Sunscreen & 10 & SPF & PF-VIS & PPF & PF-UVA & TR\% & Ivis \\
\hline I & + & 70 & 6.3 & 15.9 & 37.0 & $83.84 \%$ & 39.8 \\
\hline 2 & + & 70 & 5.8 & 14.5 & 37.8 & $82.37 \%$ & 37.9 \\
\hline 3 & + & $50+$ & 5.7 & 14.9 & 50.4 & $82.05 \%$ & 37.5 \\
\hline 4 & + & 70 & 5.1 & 12.7 & 40.3 & $79.95 \%$ & 35.1 \\
\hline 5 & + & $50+$ & 4.9 & 9.2 & 31.0 & $79.44 \%$ & 34.5 \\
\hline 6 & + & $50+$ & 4.7 & 9.5 & 24.3 & $78.50 \%$ & 33.5 \\
\hline 7 & + & 70 & 4.5 & 11.3 & 41.0 & $77.67 \%$ & 32.7 \\
\hline 8 & + & 70 & 4.3 & 10.8 & 38.6 & $76.28 \%$ & 31.2 \\
\hline 9 & + & 70 & 4.1 & 9.7 & 27.2 & $75.13 \%$ & 30.2 \\
\hline 10 & + & 60 & 3.9 & 8.2 & 23.7 & $73.98 \%$ & 29.3 \\
\hline 11 & + & 70 & 3.9 & 9.1 & 25.3 & $73.75 \%$ & 29.1 \\
\hline 12 & + & $50+$ & 3.8 & 7.6 & 25.1 & $73.49 \%$ & 28.9 \\
\hline 13 & + & 50 & 3.7 & 7.9 & 22.6 & $72.39 \%$ & 28.0 \\
\hline 14 & + & 70 & 3.7 & 8.4 & 28.8 & $72.32 \%$ & 27.9 \\
\hline 15 & + & 60 & 3.6 & 8.3 & 34.8 & $71.73 \%$ & 27.5 \\
\hline 16 & + & 50 & 3.5 & 8.0 & 19.2 & $70.60 \%$ & 26.7 \\
\hline 17 & + & 50 & 3.2 & 7.1 & 18.2 & $68.54 \%$ & 25.2 \\
\hline 18 & - & 55 & 2.0 & 5.0 & 22.3 & $48.67 \%$ & 14.8 \\
\hline 19 & - & 30 & 2.0 & 4.9 & 27.6 & $48.28 \%$ & 14.5 \\
\hline 20 & - & 30 & 1.9 & 4.8 & 20.9 & $47.86 \%$ & 14.2 \\
\hline 21 & - & 70 & 1.9 & 4.9 & 38.3 & $46.95 \%$ & 14.0 \\
\hline 22 & - & 30 & 1.9 & 4.2 & 16.0 & $45.55 \%$ & 13.3 \\
\hline 23 & - & 99 & 1.8 & 4.9 & 48.9 & $44.96 \%$ & 13.2 \\
\hline 24 & - & 50 & 1.8 & 4.5 & 19.9 & $44.94 \%$ & 13.2 \\
\hline 25 & - & 70 & 1.7 & 4.0 & 18.2 & $41.25 \%$ & 11.6 \\
\hline 26 & - & 90 & 1.7 & 4.3 & 39.0 & $38.71 \%$ & 11.1 \\
\hline 27 & - & 30 & 1.7 & 3.1 & 12.9 & $39.57 \%$ & 11.0 \\
\hline 28 & - & 80 & 1.6 & 4.0 & 33.9 & $35.88 \%$ & 9.9 \\
\hline 29 & - & 30 & 1.4 & 3.0 & 14.2 & $30.24 \%$ & 7.8 \\
\hline 30 & - & 45 & 1.2 & 2.6 & 10.2 & $15.19 \%$ & 3.6 \\
\hline 31 & - & 50 & I.I & 2.4 & 25.6 & $9.03 \%$ & 2.1 \\
\hline 32 & - & 50 & I.I & 2.4 & 25.8 & $7.63 \%$ & 1.7 \\
\hline 33 & - & 30 & 1.1 & 2.4 & 19.5 & $7.29 \%$ & 1.6 \\
\hline
\end{tabular}

After application of the product, the PMMA plate was placed in a dark room at room temperature for $30 \mathrm{~min}$ to dry the product and form a thin homogeneous film.

After the resting period, the plates were inserted into the spectrophotometric equipment for the readings (pre-irradiation). On each plate, readings were taken at six different points. The data collected by the equipment were compiled in a spreadsheet, to determine the spectrophotometric curves.

The plates were then irradiated with the Solar Simulator, again following the procedures established by standard ISO 24443. The Irradiation dose of the plates, according to the ISO 24443 , was D $\left(\mathrm{J} / \mathrm{cm}^{2}\right)=1.2 \mathrm{~J} / \mathrm{cm}^{2} \mathrm{x}$ FP-UVA (pre-irradiation).
Figure 1 shows the spectral output of the Solar Simulator used in this project and the natural sunlight as proposed in the item IV

After the irradiation period, the plates were again inserted into the spectrophotometric equipment for the post-irradiation readings.

\section{Measurement calculations}

\section{Solar VL Protection Factor (PF-VIS)}

According to our proposal, the VL Protection Factor (PFVIS) is based on the principles of the method proposed to determine the SPF in vitro and the principles of the PFUVA method according to standard ISO 24443:2012. ${ }^{26}$ 


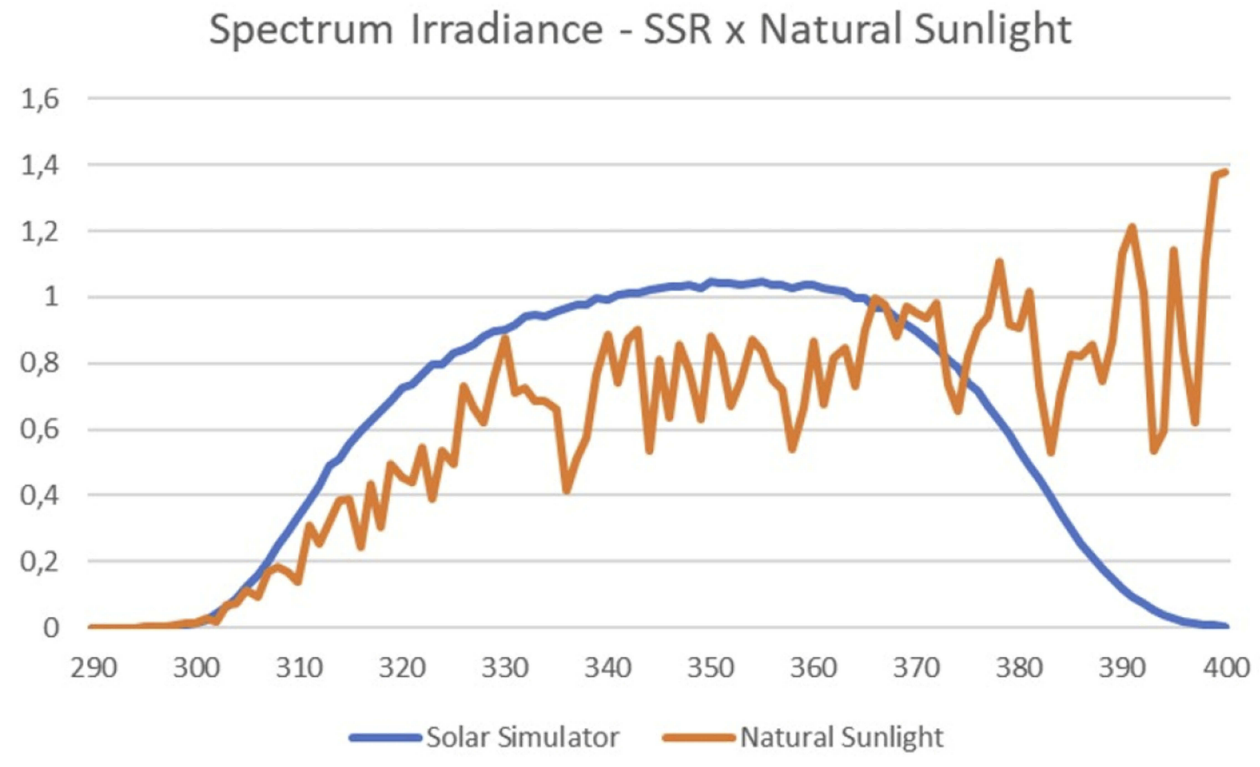

Figure I Spectral output $\left(\mathrm{W}^{*} \mathrm{~m}^{-2 *} \mathrm{~nm}^{-1}\right)$ of SSR $\mathrm{x}$ natural sunlight.

Therefore, it is necessary to determine 3 variables:

A - Absorption Spectrum of the product in the VL range $(400-450 \mathrm{~nm})$, calculated by spectrophotometric measurement, according to item IIIA.

B - UVR and VL solar spectrum for the city of São Paulo, Brazil $\left(23.3^{\circ} \mathrm{S}, 46.6^{\circ} \mathrm{W}\right)$ provided by the SBDART radiative transfer model. Calculations were performed for the summer solstice (December 21th) in the southern hemisphere at noon, detailed in item IV.

C - Spectrum of the immediate pigment darkening (IPD) proposed by Rosen (1992). ${ }^{14}$

With these three variables, the determination of the PFVIS was calculated from equation 1 :

Equation 1: PF-VIS Calculation

$$
P F-V I S=\int_{400}^{450} P(\lambda) x I(\lambda) x d(\lambda) / \int_{400}^{450} P(\lambda) x I(\lambda) \times 10^{-A(\lambda)} \mathrm{d}(\lambda)
$$

Where:

$\mathrm{P}(\lambda)$ is the IPD spectrum;

$\mathrm{I}(\lambda)$ is the radiation spectrum of the sun;

A $(\lambda)$ is the average of the monochromatic absorbance of the sunscreen after exposure to the UVR;

$\mathrm{d}(\lambda)$ is the wavelength $(1 \mathrm{~nm})$.

Integral of the absorption curve of the product in the VL range (Ivis)

As shown in our previous study ${ }^{19}$ (Schalka 2012), the integral of the area of the absorbance curve of each product, within the VL range (Ivis $400-450 \mathrm{~nm}$ ), was determined by the following equation:

Equation 2: Ivis calculation

$$
\text { Ivis }=\int_{400}^{450} \mathrm{~A}(\lambda) \mathrm{d}(\lambda)
$$

Where:

A $(\lambda)$ is the average of the monochromatic absorbance of the sunscreen after exposure to the UVR;

$\mathrm{d}(\lambda)$ is the wavelength $(1 \mathrm{~nm})$.

\section{Transmittance reduction (TR\%)}

The calculation of the percentage of transmittance reduction (\%) in the VL range, used in our previous publications, ${ }^{17,21}$ is based on the following equation:

Equation 3: TR\% Calculation

$$
T R \%(\%)=\left(1-\left(\int_{400}^{450} \operatorname{Tp}(\lambda) d(\lambda) / \int_{400}^{450} \operatorname{Tg}(\lambda) d(\lambda)\right) * 100\right.
$$

Where:

$\operatorname{Tp}(\lambda)$ is the average of the monochromatic transmittance of the sunscreen after the UVR exposure;

$\operatorname{Tg}(\lambda)$ is the average of the monochromatic glycerin transmittance (control);

$\mathrm{d}(\lambda)$ is the wavelength $(1 \mathrm{~nm})$.

Sun Protection Factor (SPF)

The labelled SPF value of the product. 


\section{UVA Protection Factor (PF-UVA)}

The PF-UVA of the product was determined using the parameters and calculations proposed in ISO 24443:2012. ${ }^{26}$

Equation 4: PF-UVA calculation

$$
P F-U V A=\int_{320}^{400} P(\lambda) x I(\lambda) x d(\lambda) / \int_{320}^{400} P(\lambda) x I(\lambda) \times 10^{-A(\lambda) * C} x d(\lambda)
$$

Where:

$\mathrm{P}(\lambda)$ is the PPD spectrum;

$\mathrm{I}(\lambda)$ is the radiation spectrum of the UVA source;

A $(\lambda)$ is the average of the monochromatic absorbance of the sunscreen after exposure to the UVR;

$\mathrm{C}$ is the coefficient of adjustment

$\mathrm{d}(\lambda)$ is the wavelength $(1 \mathrm{~nm})$.

\section{Pigmentation Protection Factor (PPF)}

The Pigment Protection Factor (PPF) was determined by associating the pigmentary response of the UVA to the VL range, as follows:

Equation 5: PPF calculation

$$
P P F=\int_{320}^{450} P(\lambda) x I(\lambda) x d(\lambda) / \int_{320}^{450} P(\lambda) x I(\lambda) \times 10^{-A(\lambda)} d(\lambda)
$$

Where:

$\mathrm{P}(\lambda)$ is the IPD spectrum;

$I(\lambda)$ is the radiation spectrum of the sun;

A $(\lambda)$ is the average of the monochromatic absorbance of the sunscreen after exposure to the UVR;

$\mathrm{d}(\lambda)$ is the wavelength $(1 \mathrm{~nm})$.

\section{Mathematical modeling of the solar radiation spectrum}

The UVR (280-400 nm) and VL (400-780 nm) solar radiation spectra were provided by the SBDART (Santa Barbara DISORT Atmospheric Radiative Transfer) ${ }^{27}$ model (Ricchazzi et al, 1998). SBDART is plane-parallel radiative transfer model based on discrete ordinate method calculations. The package is an ensemble of absorption and scattering routines for gases, water droplets, and ice crystals. In this study, we have considered the following conditions: i) clear and cloudless sky; ii) subtropical atmospheric profile; surface albedo and background aerosol properties typical for urban areas such as the city of São Paulo; iii) calculations for the summer solstice at noontime; and, iv) $0.5 \mathrm{~nm}$ spectral resolution.

\section{Statistical analysis}

The correlation between the PF-VIS and the other variables was evaluated using the Pearson correlation coefficient $\left(\mathrm{r}_{\mathrm{p}}\right)$ and the associated $P$-value. For the variables that did not present linear behavior, we calculated the Spearman $\left(\mathrm{r}_{\mathrm{s}}\right)$ correlation coefficient.

Later, we proceeded to correlate PPF with the other variables, in a similar manner.

The results were considered significant at a significance level of $5 \%(P$-value $\leq 0.05)$.

\section{Results}

The PF-VIS and the PPF were determined for the 33 evaluated products, and the results are summarized in Table 1.

\section{Solar visible light protection factor (PF- VIS)}

As shown in Table 1, the products containing iron oxide in their formulation had PF-VIS values higher than 3, whereas products without iron oxide in the formulation had PF-VIS values below 2, demonstrating that iron oxide interferes positively with the value of PF-VIS.

Initially, we compared the PF-VIS values with values of the integral area and the transmittance reduction (TR\%), both within the same wavelength range (400-450 nm), through the scatterplot between the two variables.

Figure 2 shows the scatterplot between the PF-VIS and the percentage of transmittance reduction $\mathrm{T}(\mathrm{TR} \%)$.

The chart shows a positive nonlinear relationship between the variables and, therefore, we calculated the Spearman $\left(\mathrm{r}_{\mathrm{s}}\right)$ correlation coefficient. Spearman's correlation evaluates whether there is a monotonic relationship between two continuous or discrete variables. In this type of relationship, the variables tend to change together but not necessarily at a constant rate like that found in the linear or Pearson correlation. In the PF-VIS x TR ratio, we find $r_{s}=0.9998$. The hypothesis that there is no correlation between the studied variables was tested and rejected $(p<0.001)$.

Figure 3 shows the scatterplot between the VL range and the integral of absorbance in the range of 400-450 nm (Ivis). In this case, we find $\mathrm{r}_{\mathrm{s}}=0.9998$, again the hypothesis that there is no correlation between the studied variables was tested and rejected $(p<0.001)$, showing a non-linear correlation between the two variables. 


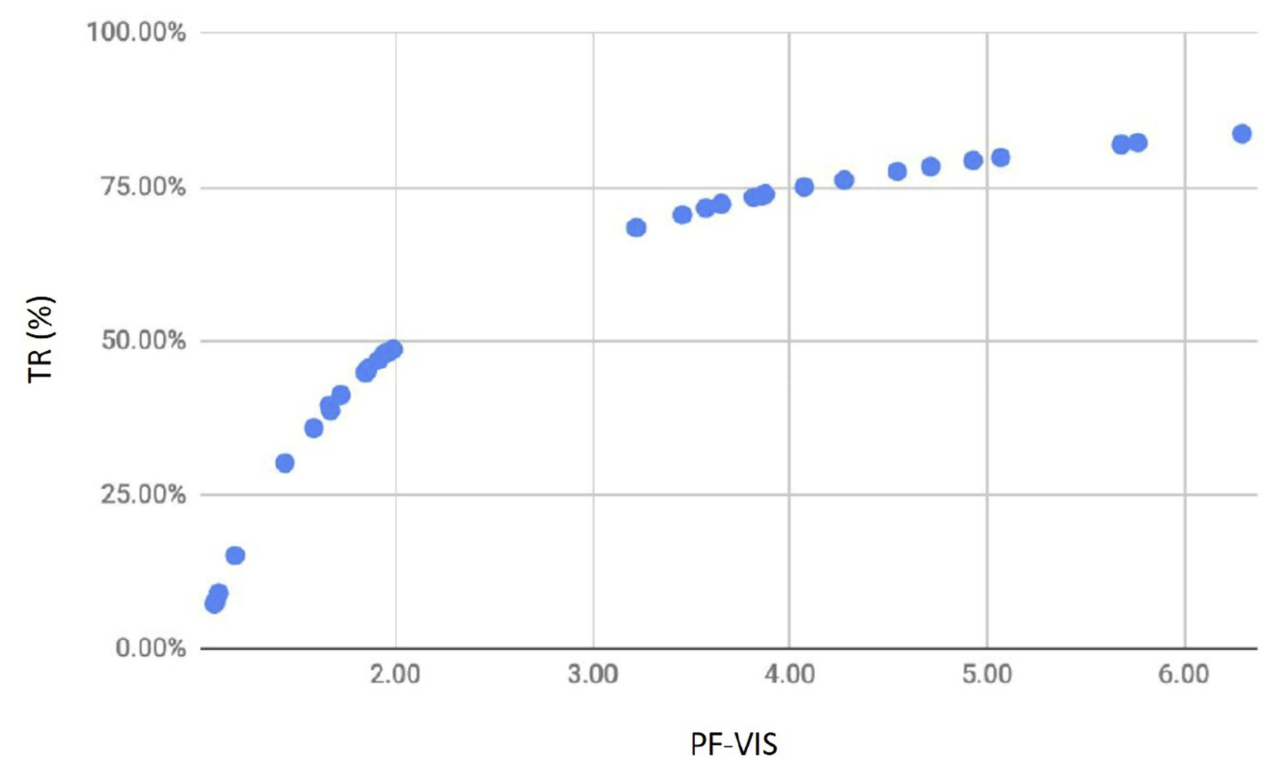

Figure 2 PF-VIS $\times$ TR (\%) scatterplot.

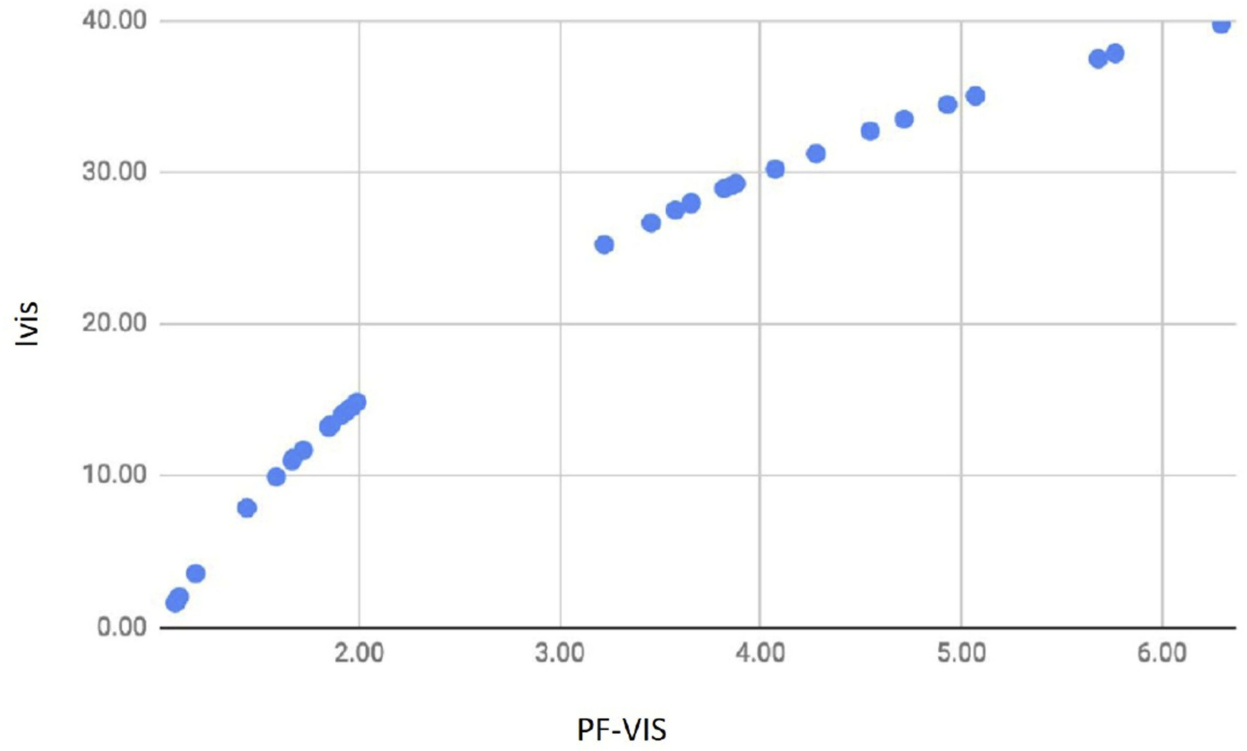

Figure 3 PF-VIS $\times$ Ivis scatterplot.

We then verified that there is a strong non-linear correlation between the PF-VIS, both with respect to the transmittance reduction (\%) and the Integral in the VL range.

We then compared the PF-VIS results with the product labelled SPF values and the PF-UVA values (determined by the ISO 24443 method), through the scatterplots between the variables.

Figure 4 shows the scatterplot between the PF-VIS and SPF values. The hypothesis that there is no correlation between the studied variables was tested and not rejected, and indicates that there is no statistical significance for the correlation between PF-VIS and SPF $\quad\left(r_{p}=0.2817\right.$; $P$-value $=0.1725)$ :

Figure 5 shows the scatterplot between PF-VIS and PFUVA values. Again, the hypothesis that there is no correlation between the studied variables was tested and rejected, concluding that the correlation between the evaluated values $\left(\mathrm{r}_{\mathrm{p}}=0.5296 ; P\right.$-value $\left.=0.0064\right)$ was statistically significant. We then verified that there is a weak linear correlation between PF-VIS and PF-UVA. 


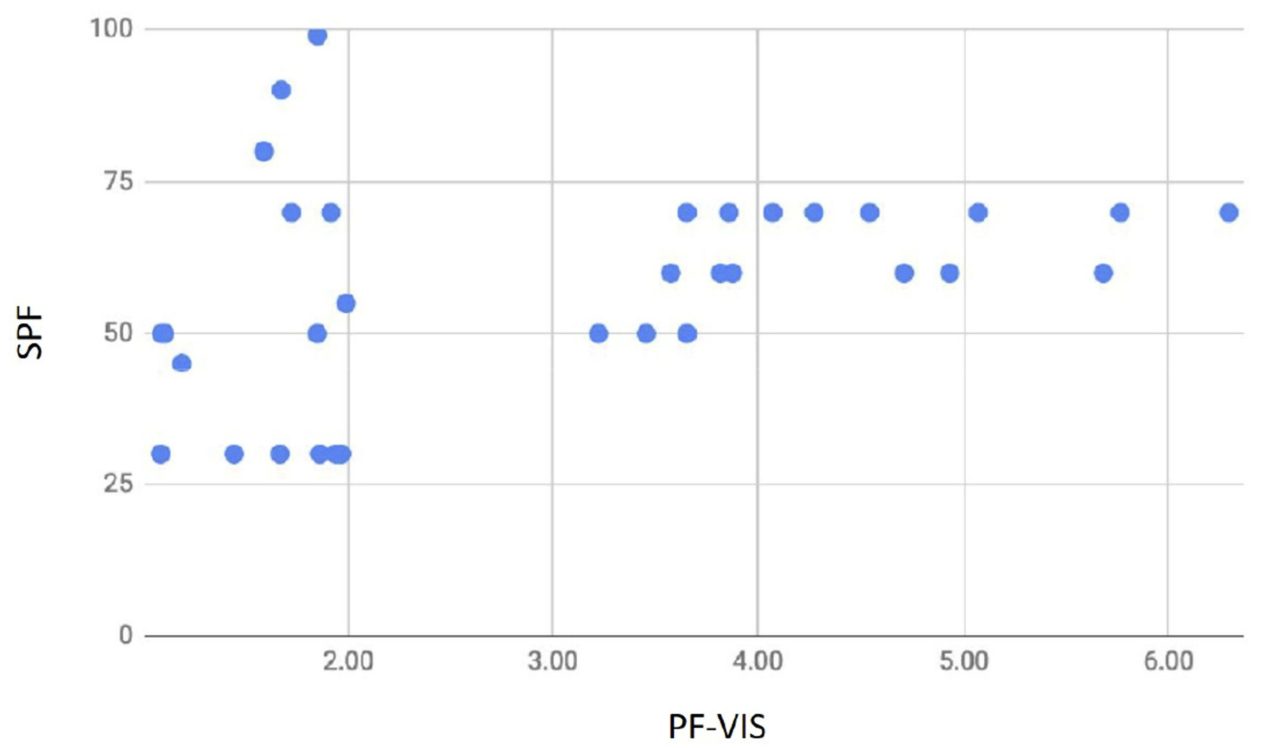

Figure 4 PF-VIS $\times$ labelled SPF scatterplot.

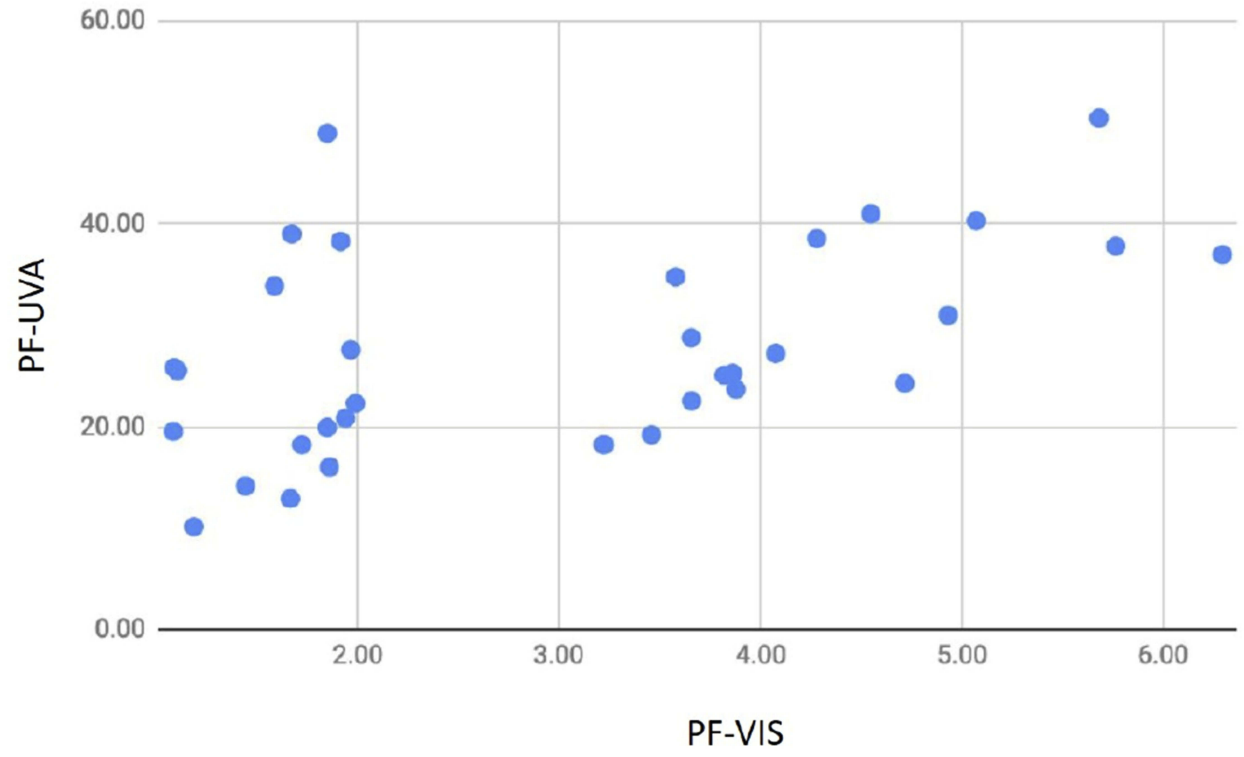

Figure 5 PF-VIS $\times$ PF-UVA scatterplot.

\section{Pigment protection factor (PPF)}

As shown in Table 1, the products containing iron oxide in their formulation have PPF higher than 7, whereas those without iron oxide in their formulation have PPF values less than 5 . This shows that iron oxide has positive impact on the pigmentation protection factor due to its action in the long UVA and VL range.

We compared the PPF values with the PF-VIS, SPF and PF-UVA values through the scatterplots between the variables. Figure 6 shows the very strong positive correlation between the PF-VIS and PPF variable $\left(r_{p}=0.9081\right)$. The hypothesis that there is no correlation between the studied variables was tested and rejected $(P$-value $<0.001)$. On the other hand, as shown in Figure 7, the positive correlation between the PPF and the SPF is weak $\left(r_{p}=0.398\right)$, but is statistically significant, since the hypothesis that there is no correlation between the studied variables was tested and rejected $(P$-value $=0.0217)$.

Finally, the scatterplot between the PPF and PF-UVA variables, shown in Figure 8, shows a moderate positive 


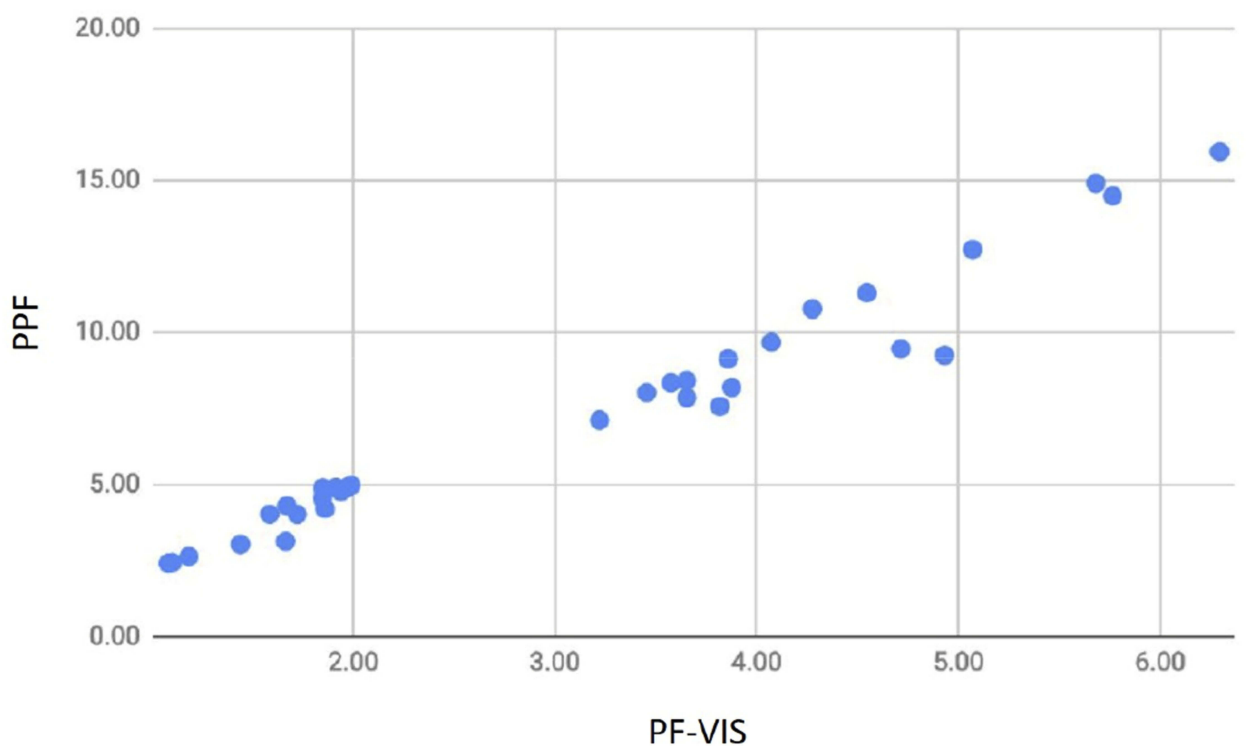

Figure 6 PF-VIS $\times$ PPF scatterplot.

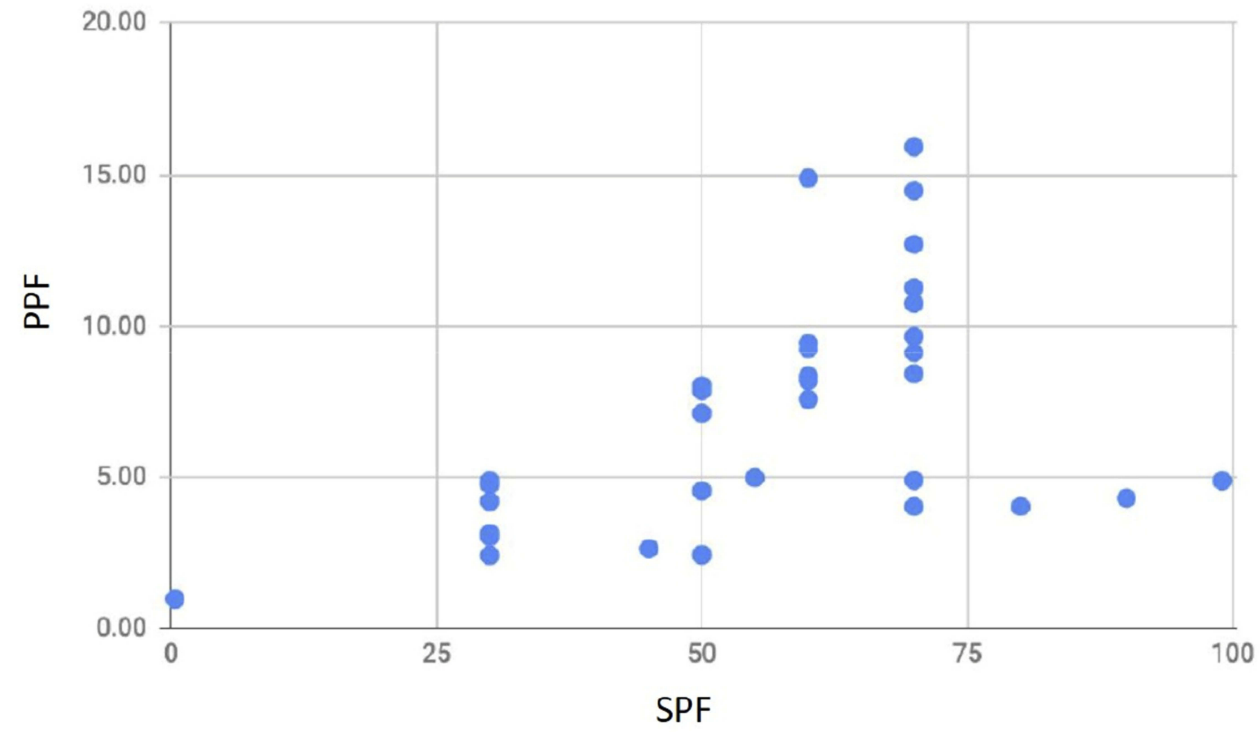

Figure 7 PPF vs SPF scatterplot.

correlation $\left(r_{p}=0.5418\right)$; again the hypothesis that there is no correlation between the variables was tested and rejected $(P$-value $<0.001)$.

\section{Discussion}

Our study proposed using the model created by Sayre et $\mathrm{al}^{25}$ to determine the photoprotective efficacy in the visible light range. We chose to use only the high energy VL range (blue light), as Rosen et $\mathrm{al}^{14}$, in determining the IPD spectrum in their study, observed that the VL pigmentation potential would occur only at wavelengths below $470 \mathrm{~nm}$. In addition, instead of using the spectrum of the light-emitting equipment, we chose to use the solar spectrum estimated for the city of São Paulo (Brazil), at noon, on the summer solstice of the Southern Hemisphere (December 21st). The city of Sao Paulo, the tenth largest city in the world with about 20 million inhabitants, is located in the tropic of Capricorn and, therefore, is the closest point to the Sun at the summer solstice.

Our results showed that the sunscreens with the presence of iron oxide had PF-VIS values between 6.29 and 3.22 , higher than the values found in sunscreens without 


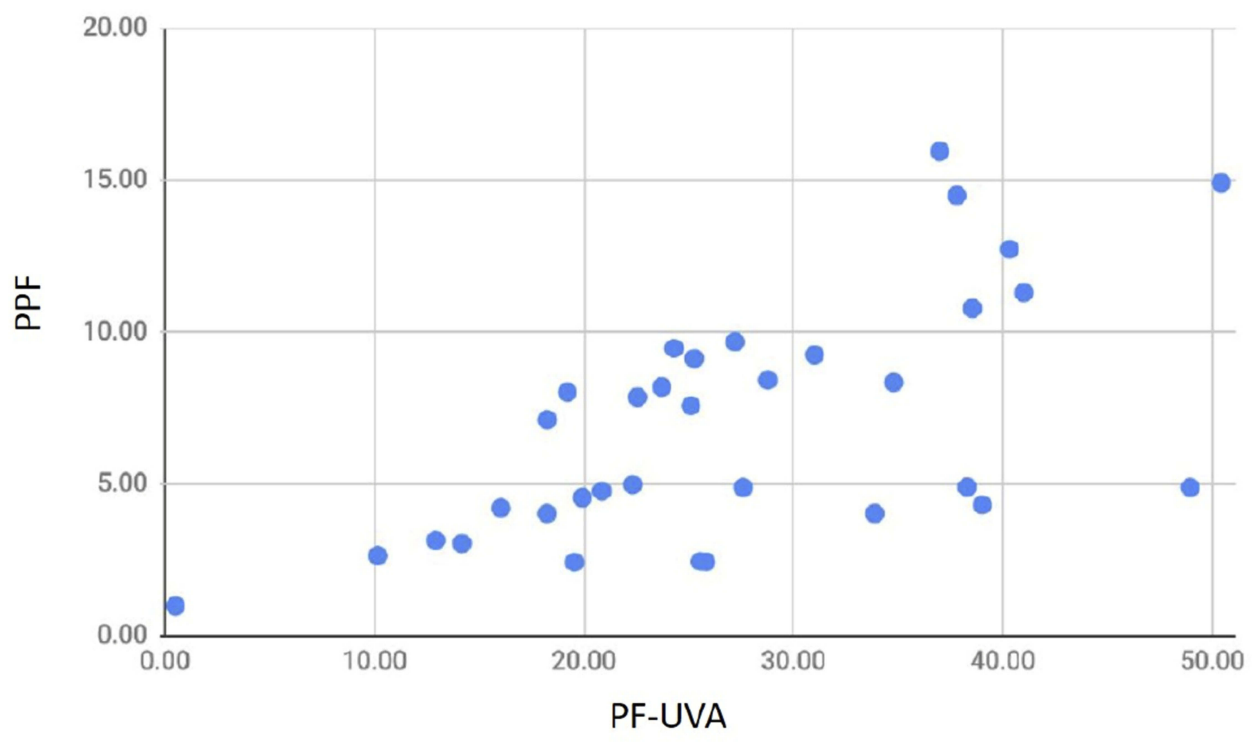

Figure 8 PPF $x$ PF-UVA scatterplot.

iron oxide (PF-VIS between 1.99 and 1.08). In addition, we identified certain PF-VIS ranges clearly related to the increase in the value of the integral in the VL range and to the increase of the transmission percentage, which leads us to propose a new classification of sunscreens per range of protection against VL.

In this proposal products having at least PF-VIS greater than 2 and a transmittance reduction (\%) greater than 60 would only be classified as having some level of protection against VL. Among the products with some level of protection against the VL, four categories are proposed, as indicated in Table 2.

Comparing the PF-VIS values with the other measures of protection against VL (Ivis and TR(\%)), we observed a strong non-linear correlation between them. This indicates that the increase of the PF-VIS is related to the increase of the other measures of protection determination in this range, but with a trend to stabilize the greater values, being therefore non-linear (logarithmic).
When comparing the PF-VIS results with the SPF values, we observed that there is no correlation between the two measurements. That is, products with higher SPF values do not necessarily provide greater protection against VL, since this is expected, since the SPF is a measure of protection against sunburn, a biological event essentially related to short UVB and UVA and consistent with our first study, ${ }^{17}$ where we also did not observe a correlation.

Regarding the comparison between PF-VIS and PFUVA, we identified a weak correlation. This data is unprecedented in the literature and shows that there is a trend for products with greater UVA protection to provide greater protection in the VL range, possibly due to the increase of inorganic filters. As the correlation is considered weak, the choice of sunscreens for protection against the VL should not be based on the PF-UVA value.

In the second part of our study, we decided to extend the analysis of the sunscreens evaluated for the entire IPD

Table 2 Proposed classification of sunscreens by range of protection against visible light

\begin{tabular}{|c|c|c|c|}
\hline Protection against VL & Stars & PF-VIS & Transmittance reduction (\%TR) \\
\hline Very High & & $>5.0$ & \multirow[t]{4}{*}{$>60 \%$} \\
\hline High & & $4.1-5.0$ & \\
\hline Medium & & $3.1-4.0$ & \\
\hline Weak & & $2.1-3.0$ & \\
\hline Absent & & $<2.0$ & $<60 \%$ \\
\hline
\end{tabular}


spectrum as defined by Rosen ${ }^{14}$, including the UVA and high energy VL range, from 320 to $450 \mathrm{~nm}$.

As the use of sunscreens with protection against VL is closely related to the protection against pigmentary diseases, it seems reasonable to do this evaluation considering the entire spectrum of pigmentation, as this may provide relevant data in the categorization of sunscreens for this purpose. Thus, we determined the pigmentation protection factor (PPF) using the same principles of PF-VIS, but with the increase of the spectrum for the UVA range.

Thus, we observed that products containing pigments offered the greatest protection, with PPF higher than 7 (15.95 and 7.11) and products without iron oxide presented values lower than 5 (4.98-2.42). These values are higher than those found for the PF-VIS, since the protection offered in the UVA range is even more efficient than the protection offered against the VL.

When comparing the PF-VIS and PPF results for the 33 products studied, we observed that there is a strong positive correlation between the variables, with a correlation index of 0.91, showing that products with higher PF-VIS also have higher PPF. However, comparing the PPF and the SPF and the PF-UVA, correlation indices are low. That is, when evaluating a sunscreen by the SPF or PF-UVA value, we cannot infer protection against pigmentation, with the PF-VIS and the PPF evaluation being more suitable.

\section{Conclusion}

As reported by other studies, this study was able to demonstrate that the use of spectrophotometric measurements can be an important parameter for evaluating photoprotective efficacy in the VL range (mainly Blue light or High Energy Visible Light) of topical sunscreen products.

The use of a quantitative metrics for determining the solar VL protection factor, in the already established concepts for determining the SPF and the PF-UVA in vitro was adequate as a measure of the effectiveness of sunscreens in the visible light range.

The novelty of the method proposed is the choice of the range of visible light evaluated in the IPD spectrum and the source spectrum. Validation of the method, with a greater range of products and a correlation with biological endpoints is needed.

The use of a classification table for sunscreens, based on the PF-VIS value and transmittance reduction, can be an interesting tool for choosing sunscreens for users and healthcare professionals.
In addition to the PF-VIS, this study also proposes new metrics for evaluating sunscreens against the pigmentary effect of solar radiation (including the UVA and the VL), appearing as an adequate alternative in identifying the efficacy of sunscreens in the prevention of pigmented dermatoses, such as melasma and post inflammatory pigmentation.

\section{Disclosure}

The authors report no conflicts of interest in this work.

\section{References}

1. Schalka S, Steiner D, FN R, et al. Brazilian consensus on photoprotection. An Bras Dermatol. 2014;89(6suppl 1):1-74. doi:10.1590/ abd1806-4841.20143971

2. Mahmoud BH, Hexsel CL, Hamzavi IL, Lim HW. Effects of visible light on the skin. Photochem Photobiol. 2008;84:450-462. doi:10.1111/j.1751-1097.2007.00286.x

3. Liebel F, Kaur S, Ruvolo E, Kollias N, Southall MD. Irradiation of skin with visible light induces reactive oxygen species and matrixdegrading enzymes. J Invest Dermatol. 2012;132(7):1901-1907. doi:10.1038/jid.2011.476

4. Botto NC, Warshaw EM. Solar urticaria. J Am Acad Dermatol. 2008;59(6):909-920. doi:10.1016/j.jaad.2008.08.020

5. Hawk JL. Chronic actinic dermatitis. The British Photodermatology Group. Photodermatol Photoimmunol Photomed. 2004;20(6):312314. doi:10.1111/j.1600-0781.2004.00129.x

6. Murphy GM. The cutaneous porphyrias: a review. Br J Dermatol. 1999;140(4):573-581. doi:10.1046/j.1365-2133.1999.02754.x

7. Kollias N, Baqer A. An experimental study of the changes in pigmentation in human skin in vivo with visible and near infrared light. Photochem Photobiol. 1984;39(5):651-659.

8. Ramasubramaniam R, Roy A, Sharma B, Nagalakshmi S. Are there mechanistic differences between ultraviolet and visible radiation induced skin pigmentation? Photochem Photobiol Sci. 2011;10:1887-1893. doi:10.1039/c1pp05202k

9. Mahmoud BH, Ruvolo E, Hexsel CL, et al. Impact of long-wavelength UVA and visible light on melanocompetent skin. $J$ Invest Dermatol. 2010;130(8):2092-2097. doi:10.1038/jid.2010.95

10. Randhawa M, Seo I, Liebel F, Southall MD, Kollias N, Ruvolo E. Visible light induces melanogenesis in human skin through a photoadaptive response. PLoS One. 2015. doi:10.1371/journal.pone.0130949

11. Pathak MA, Riley FC, Fitzpatrick TB. Melanogenesis in human skin following exposure to long-wave ultraviolet and visible light. $J$ Invest Dermatol. 1962;39:435-443. doi:10.1038/jid.1962.136

12. Duteil L, Cardot-Leccia N, Queille-Roussel C, et al. Differences in visible light-induced pigmentation according to wavelengths: a clinical and histological study in comparison with UVB exposure. Pigment Cell Melanoma Res. 2014;27(5):822-826. doi:10.1111/pcmr.12273

13. Porges SB, Kaidbey KH, Grove GL. Quantification of visible lightinduced melanogenesis in human skin. Photodermatol. 1988;5 (5):197-200.

14. Rosen CF, Jacques SL, Stuart ME, Gange RW. Immediate pigment darkening visual and reflectance spectrophotometric analysis of action spectrum. Photochem Photobiol. 1990;51(5):583-588.

15. Kohli I, Chaowattanapanit S, Mohammad TF, et al. Synergistic effects of long-wavelength ultraviolet A1 and visible light on pigmentation and erythema. Br J Dermatol. 2018;178(5):1173-1180. doi:10.1111/bjd. 15940

16. Boukari F, Jourdan E, Fontas E, et al. Prevention of melasma relapses with sunscreen combining protection against $\mathrm{UV}$ and short wavelengths of visible light: a prospective randomized comparative trial. $J$ Am Acad Dermatol. 2015;72(1):189-90.e1. doi:10.1016/j.jaad.2014.08.023 
17. Martini APM, Maia Campos PMBG. Influence of visible light on cutaneous hyperchromias: clinical efficacy of broad-spectrum sunscreens. Photodermatol Photoimmunol Photomed. 2018;34(4):241248. doi:10.1111/phpp. 12377

18. Castanedo-Cazares JP, Hernandez-Blanco D, Carlos-Ortega B, Fuentes-Ahumada C, Torres-Alvarez B. Near-visible light and UV photoprotection in the treatment of melasma: a double-blind randomized trial. Photodermatol Photoimmunol Photomed. 2014;30(1):3542. doi:10.1111/phpp.12086

19. Schalka S, Addor FAS, Agelune CM, Pereira VMC. Sunscreen protection against visible light: a new proposal for evaluation. Surg Cosmet Dermatol. 2012;3(4):45-52.

20. Osterwalder U, Sohn M, Herzog B. Global state of sunscreens. Photodermatol Photoimmunol Photomed. 2014;30(2-3):62-80.

21. Diffey BL, Faar PM. Sunscreeen protection against UVB, UVA and blue light: an in vivo and in vitro comparision. $\mathrm{Br} J$ Dermatol. 1991;124:258-263. doi:10.1111/j.1365-2133.1991.tb0 0570.x
22. Kaye ET, Levin JA, Blank IH, Arndt KA, Anderson RR. Efficiency of opaque photoprotective agents in the visible light range. Arch Dermatol. 1991;127:351-355.

23. Schalka S. New data on hyperpigmentation disorders. J Eur Acad Dermatol Venereol. 2017;31 Suppl 5:18-21. doi:10.1111/jdv.14411

24. Ruvolo E, Fair M, Hutson A, Liebel F. Photoprotection against visible light-induced pigmentation. Int J Cosmet Sci. 2018;40 (6):589-595. doi:10.1111/ics.12502

25. Sayre RM, Agin PP, LeVee GJ, Marlowe E. A comparison of in vivo and in vitro testing of sunscreening formulas. Photochem Photobiol. 1979;29(3):559-566.

26. International Standards Organization, Cosmetics -Sun protection test methods- Determination of sunscreen UVA photoprotection in vitro. ISO 24443; 2012. doi:10.1094/PDIS-11-11-0999-PDN

27. Ricchazzi P, Yang SR, Gautier C, Sowle D. SBDART: A research and teaching software tool for Plane-parallell radiative transfer in the earth's atmosphere. Bull Am Meteorol Soc. 1998;79(10):2101-2114. doi:10.1175/1520-0477(1998)079<2101:SARATS>2.0.CO;2

\section{Publish your work in this journal}

Clinical, Cosmetic and Investigational Dermatology is an international, peer-reviewed, open access, online journal that focuses on the latest clinical and experimental research in all aspects of skin disease and cosmetic interventions. This journal is indexed on CAS.
The manuscript management system is completely online and includes a very quick and fair peer-review system, which is all easy to use. Visit http://www.dovepress.com/testimonials.php to read real quotes from published authors. 